\title{
NOTE ON A PARTITION THEOREM
}

\author{
by GEORGE E. ANDREWS $\dagger$
}

(Received 17 February, 1969)

1. Introduction. In $[1$, p. 130$]$ the following partition theorem was deduced from a general theorem concerning the limit of a recurrent sequence.

THEOREM. Let $r \geqq 2$ be an integer. Let $P_{1}(n)$ denote the number of partitions of $n$ into parts that are either even and not congruent to $4 r-2(\bmod 4 r)$ or odd and congruent to $2 r-1$, $4 r-1(\bmod 4 r)$. Let $P_{2}(n)$ denote the number of partitions of $n$ of the form $n=b_{1}+\ldots+b_{s}$, where $b_{i} \geqq b_{i+1}$, and for $b_{i}$ odd, $b_{i}-b_{i+1} \geqq 2 r-1 \quad\left(1 \leqq i \leqq s\right.$, where $\left.b_{s+1}=0\right)$. Then $P_{1}(n)=P_{2}(n)$.

Now considering the generating function for $P_{1}(n)$, we have

$$
\begin{aligned}
1+\sum_{n=1}^{\infty} P_{1}(n) q^{n} & =\prod_{j=1}^{\infty}\left(1-q^{4 r j-2}\right)\left(1-q^{2 j}\right)^{-1}\left(1-q^{2 r j-1}\right)^{-1} \\
& =\prod_{j=1}^{\infty}\left(1+q^{2 r j-1}\right)\left(1-q^{2 j}\right)^{-1} \\
& \equiv 1+\sum_{n=1}^{\infty} P_{3}(n) q^{n}
\end{aligned}
$$

where $P_{3}(n)$ is the number of partitions of $n$ into parts that are either even or else congruent to $2 r-1(\bmod 2 r)$ with the further restriction that only even parts may be repeated. Thus $P_{1}(n)=P_{3}(n)$.

The object of this note is to provide a simple combinatorial proof of the fact that $P_{2}(n)=$ $P_{3}(n)$; equation (1) then yields the above theorem.

2. Proof that $P_{2}(n)=P_{3}(n)$. We provide a one-to-one correspondence between the sets of partitions to be counted.

Let $\pi$ be a partition of the type enumerated by $P_{2}(n)$. Then represent $\pi$ graphically with each even part $2 m$ represented by two rows of $m$ nodes and each odd part $2 m+1$ represented by two rows of $m+1$ nodes and $m$ nodes respectively. For example, $11+4$ becomes

Now read the graph vertically with the proviso that $r$ columns are always to be grouped as a single part whenever the lowest node in the most right-hand column of the group comes from what was originally the right-hand-most node contributed by an odd part. Thus in our

† Partially supported by NSF Grant GP 8075. 
example with $r=2$, we obtain in this manner the partition $4+4+2+2+3$. Now since the condition on partitions enumerated by $P_{2}(n)$ is $b_{i}-b_{i+1} \geqq 2 r-1$ whenever $b_{i}$ is odd, we see that our groupings of $r$ columns always have one less node than a rectangle of $r \times 2 v$ nodes; thus a part congruent to $2 r-1(\bmod 2 r)$ is produced. Since originally odd parts were distinct, we see that now odd parts will be congruent to $2 r-1(\bmod 2 r)$ and will not be repeated, and since originally all odd parts were greater or equal to $2 r-1$, we see that there will always be $r$ columns available for each grouping. Thus in this way we have produced a partition of the type enumerated by $P_{3}(n)$. Clearly our correspondence is one-to-one into; however, the above process is reversible and thus the correspondence is onto. Hence $P_{2}(n)=P_{3}(n)$.

As an example we take $r=2, n=11$. The corresponding partitions are listed opposite each other in the following table

\begin{tabular}{ll}
\multicolumn{1}{c}{$P_{2}$} & \multicolumn{1}{c}{$P_{3}$} \\
11 & $2+2+2+2+3$ \\
$9+2$ & $4+2+2+3$ \\
$8+3$ & $7+2+2$ \\
$7+4$ & $4+4+3$ \\
$7+2+2$ & $6+2+3$ \\
$6+5$ & $7+4$ \\
$5+2+2+2$ & $8+3$ \\
$4+4+3$ & 11
\end{tabular}

\section{REFERENCES}

1. G. E. Andrews, On Schur's second partition theorem, Glasgow Math. J. 8 (1967), 127-132.

The Pennsylvania State University

University Park, Pennsylvania 16802 